\title{
Potencialidades e limites da aplicação do monitoramento comunitário nas unidades de conservação da Amazônia: uma reflexão a partir do ProBUC
}

\author{
Potentialities and limits upon the application of community monitoring on Amazon's conservation units: a reflection from \\ ProBUC.
}

\author{
Daniel Carneiro Costa', Guillaume Antoine Marchand ${ }^{2}$ \\ ' Graduado em Economia, Universidade Federal do Amazonas, Manaus, Brasil. \\ ${ }^{2}$ Graduado em Historia Antiga, Universidade dAvignon et des Pays de Vaucluse,
}

\section{Resumo}

O ProBUC - programa de monitoramento da biodiversidade e do uso sustentável de recursos naturais em unidades de conservação estaduais do Amazonas - é uma inovação no processo de monitoramento comunitário dentro da Amazônia. Ele é, em boa medida, reflexo do processo de avaliação e gestão de áreas protegidas mundiais. Sua finalidade é a de subsidiar a gestão destas unidades de conservação por meio de informações obtidas pelo monitoramento exercido pelas próprias comunidades locais. Neste trabalho, são expostos os detalhes metodológicos de funcionamento e os objetivos do programa amazonense conforme a descrição oficial e os locais de implantação, que se referem às três unidades de conservação em que está implementado. Apresentam-se os aspectos que antecedem e inspiram a criação do ProBUC, partindo-se do referencial teórico que discute do monitoramento convencional até o surgimento da proposta do monitoramento comunitário em países tropicais, enfatizando-se as limitações de ambas as modalidades. Num segundo momento, as limitações e potencialidades deste programa amazonense são expostas dentro de uma avaliação crítica de seu funcionamento. Esta análise decorre de leituras de autores internacionais que discutem a problemática do monitoramento comunitário, de observações diretas feitas em trabalho de campo e de entrevistas com comunitários das unidades de conservação em que o programa em questão está estabelecido. Para esta fundamentação também contribuiu a participação em reunião técnica de avaliação do ProBUC realizada por sua equipe técnica no ano de 2013. Constata-se que a viabilidade deste programa depende da superação de importantes desafios como a dependência financeira de instituição internacional, a ausência de uma equipe técnica permanente, a enorme lacuna existente no tratamento de dados coletados, a participação restrita dos comunitários no desenvolvimento diário do programa, dentre outros. Estas limitações fazem com que o trabalho realizado pelos moradores locais não esteja situado dentro de uma estratégia governamental de desenvolvimento local. Ademais, os aspectos adaptativo e participativo do programa são potenciais que precisam ser valorizados para seu aperfeiçoamento. Conclui-se que se faz urgente que o governo estadual se aproprie deste programa e o dote das condições necessárias para que possa ser realmente assumido pelas comunidades e incentive a proliferação de outras iniciativas regionais semelhantes. A construção do ProBUC, neste sentido, seria de grande valia para a consolidação de alternativas de monitoramento comunitário no complexo contexto amazônico.

Palavras-chaves: Monitoramento comunitário; Gestão de unidades de conservação, Participação comunitária

\begin{abstract}
The ProBUC - monitoring program of biodiversity and the sustainable use of natural resources in protected areas of the Amazonas state - is an innovation in the community monitoring process within the Amazon. It is, in large measure, a consequence of the evaluation process and management of protected areas worldwide. Its purpose is to support the management of these protected areas through information obtained by local communities monitoring. In this work, we exposed the methodological details of the operating system of the program and its objectives as the official description made by the government of the Amazonas and the way it was implemented three protected areas. First, the aspects that precede and inspire the creation of ProBUC are presented, starting from the theoretical framework that discusses the conventional monitoring until the appearance of the proposed community monitoring in tropical countries, emphasizing the limitations of both modalities. Secondly, the limitations and potentials of this Amazon program are exposed within a critical evaluation of its functioning. This analysis stems from readings of international authors who discuss the issue of community monitoring direct observations made during field works and interviews with inhabitants of the conservation units within the program in question was established. Our analysis is also reinforced by taking part in in technical review meeting held by the ProBUC's crew in 2013. It was noted that the viability of this program depends on overcoming key challenges such as financial dependence on international institution, the absence of a permanent technical staff, the huge gap in the treatment of data collected, the limited participation of the community in the daily development of the program-, among others. These limitations mean that the work done by local inhabitants is not located within a government strategy for local development. Furthermore, adaptive and participatory aspects of the program are potentials that need to be valued for its improvement. We conclude that it is very urgent that the state government take ownership of this program and equipped it with the necessary means in order to see its appropriation by the local communities and to encourage the proliferation of other similar regional initiatives-. The construction of ProBUC in this way would be of great value to the consolidation of alternative community monitoring in the complex Amazonian context.
\end{abstract}

Keywords: Community monitoring; Management of protected areas, Community Participation 


\section{INTRODUÇÃO}

O envolvimento de comunidades locais na gestão e avaliação de áreas protegidas que apresentam rica biodiversidade ganhou força nas últimas décadas. Neste sentido, o monitoramento exercido diretamente por elas com a devida orientação científica também se tornou uma atividade valorizada. Segundo Abbot e Guijit (1998, p. 97) "[...] esta forma alternativa de monitoramento consiste em envolver nesta execução diretamente gestores e comunitários que vivem em meio à biodiversidade [...]". Para Evans e Guariguata (2008), estes programas de monitoramento de cunho comunitário se prestam, principalmente, a subsidiar as gestões de florestas articuladas aos meios de subsistência e bem estar das comunidades locais, conservação da biodiversidade e envolvimento de instituições locais nos processos decisórios. Este auxílio se desenvolveria, conforme STUART HILL et al (2005), pelo uso das informações para tomar decisões que irão impactar os rumos da gestão da área preservada em que se inserem.

Na Amazônia, porém, a adoção de métodos de gestão e avaliação das unidades de conservação (UCs) alheias às especificidades das realidades locais resultou em certa ineficiência. A adoção do monitoramento comunitário exigia a criação de um programa dotado de adaptação e flexibilidade, capaz de lidar tanto com a diversidade ecossistêmica quanto social existente (DAVIES, 1998). Métodos alternativos como o Sistema de Indicadores Socioambientais para as unidades de conservação da Amazônia Brasileira (SISUC) representam tentativas de criação de sistemas de avaliação e monitoramento da gestão das UCs localizadas nesta região brasileira a partir das experiências locais e dentro de uma metodologia de construção coletiva e participativa. O ProBUC, nesse contexto, surge com a pretensão de ser uma resposta a este vazio dentro da realidade amazonense. Trata-se de uma inovação do governo estadual que visa ser estendida a todo o sistema estadual de unidades de conservação, tendo como proposta o monitoramento diretamente efetuado pelas comunidades locais. No presente, está implementado o ProBUC em três unidades de conservação: as reservas de desenvolvimento sustentável do Uatumã e Uacari e no parque estadual do Rio Negro Setor Norte.

O ProBUC, como programa de monitoramento da biodiversidade e do uso de recursos naturais com metodologia participativa, está, conforme Junior et al (2011, p.7), numa fase de consolidação. Vive um momento importante para identificar seus avanços e limitações nas UCs em que se estabeleceu. Neste artigo é feita uma reflexão sobre os limites e potencialidades deste programa amazonense baseado no debate internacional da temática do monitoramento comunitário, observações de campo e entrevistas efetivadas com comunitários que vivem em UCs onde se insere o programa, incluindo a reunião técnica de avaliação de sua gestão. Constata-se que sua gestão precisa ser modificada profundamente para realmente auxiliar a gestão das UCs como almejado.

Esta pesquisa foi desenvolvida para verificar até que ponto o programa ProBUC corresponde às condições e características de um efetivo programa de monitoramento comunitário, não obstante as peculiaridades inerentes à realidade amazônica, sobretudo no que se refere à participação direta das comunidades locais. As atividades de campo que envolveram as entrevistas, inclusive, permitiram contrastar a realidade encontrada com a propaganda oficial acerca de como se processa o ProBUC.

\section{METODOLOGIA}

\subsection{Objeto de estudo e sua localização}

A criação do ProBUC corresponde a uma estratégia do governo estadual de expandir o monitoramento comunitário a todo o sistema estadual de unidades de conservação (SEUC), atualmente composto por 41 unidades de conservação (UCs). O monitoramento executado pelo ProBUC apresenta oficialmente 06 componentes, que correspondem aos recursos e espécies a serem monitorados: fauna, trânsito de embarcações, quelônios aquáticos, recenseamento, pesca comercial e jacarés. O monitoramento da fauna diz respeito à observação e registro da diversidade de animais silvestres, incluindo raridades, em trilhas pré-definidas que são percorridas pelo monitor responsável. O de trânsito de embarcações menciona a anotação da entrada e saída de embarcações no interior da UC. O de quelônios aquáticos se relaciona ao controle e soltura de quelônios nas praias das comunidades, registrando-se sua variedade. $\mathrm{O}$ de recenseamento é realizado a partir de visitas semanais que o monitor faz a uma quantidade determinada de comunitários com o objetivo de saber o que cada família produz, pesca e 
caça. A pesca comercial não foi encontrada nas viagens ocorridas, mas se refere ao pescado comercializado pelos comunitários e o de jacarés tem a ver com o controle por meio de contagem de ovos de jacarés que são depositados nas praias comunitárias das UCs.

Entre 2005 e 2010, este programa foi implantado em três unidades de conservação, sendo duas unidades de uso sustentável, que são as Reservas de Desenvolvimento Sustentável do Uatumã e do Uacari, e em uma unidade de Proteção Integral, o Parque Estadual do Rio Negro Setor Norte. Conforme a divulgação oficial, os monitores seriam escolhidos democraticamente em reuniões com todos os comunitários de cada uma das UCs envolvidas. Na reserva do Uacari, que se localiza no rio Juruá, município de Carauari, o ProBUC está implantado desde 2005, com a participação do Instituto Chico Mendes (ICMBio) e da Universidade Federal do Amazonas, tendo o aporte financeiro da Fundação Moore, por meio do Programa "Áreas Protegidas da Amazônia (ARPA)" e do Projeto Corredores Ecológicos. De acordo com Batista et al (2008, p.117) possui uma área de 633.000 hectares, sendo habitada aproximadamente por 236 famílias localizadas em 32 comunidades. Na Reserva do Uatumã e no Parque Rio Negro Setor Norte este Programa está em implantação desde 2008. Na Reserva Uatumã apresentava, por meio de seu plano de gestão elaborado em 2007, cerca de 257 famílias. Nesta unidade de conservação, que envolve os municípios de Itapiranga e São Sebastião do Uatumã e tem 424.430 hectares, o ProBUC é implementado em parceria com o Instituto de Conservação e Desenvolvimento Sustentável do Amazonas (IDESAM) e com o Centro de Pesquisas e Proteção de Quelônios Aquáticos (CPPQA). No Parque Estadual Rio Negro Setor Norte, localizado na margem direita do rio Negro, no município de Noivo Airão, com uma área de 146028 hectares, vivem aproximadamente 230 famílias. Nesta localidade, o programa é desenvolvido em parceria com a Fundação Vitória Amazônica, como informa Borges et al (2008, p. 16).

Há de se destacar que a inserção do monitoramento comunitário permite localizar, conforme a propaganda governamental, as oportunidades e potencialidades a serem ajustadas ao plano de manejo da unidade de conservação, permitindo, assim, aproveitamento econômico e familiar dos comunitários por meio do manejo de espécies aptas. Esta atividade participativa requer mobilização e sensibilização de todos comunitários, contribuindo para o fortalecimento de seus vínculos e, por conseguinte, viabilizando sua influência na condução da gestão da unidade de conservação. De acordo com Junior et al (2011, p.13), este trabalho do monitoramento comunitário possibilita:

Empoderamento das comunidades para atuação mais participativa na gestão da UC;

Valorização do conhecimento e dos modos de vida tradicionais;

Integração entre o conhecimento científico e o conhecimento tradicional;

Geração de benefícios diretos aos comunitários monitores;

Capacitação técnica dos comunitários;

Sensibilização sobre questões ambientais;

Redução de custos para a realização das atividades de monitoramento.

Coordenado por uma equipe técnica, o PrOBUC, inicialmente, prevê a realização de um curso de dois módulos com os monitores (nivelamento e especialização), onde os conhecimentos tradicionais são associados aos formulários e à metodologia das atividades de campo (JUNIOR, et al, 2011, p. 34). O monitoramento efetuado pelos comunitários é também monitorado. O CEUC possui uma metodologia chamada "ciclo PDCA" P - P (plan) planejar; D (do) executar; C (check) checar; A (act) agir corretivamente. Esta metodologia consiste em checar os dados provenientes das atividades comunitárias, ajustá-las e reencaminhá-las para serem socializadas e discutidas em fóruns com os comunitários, gestores e pesquisadores do Programa. Neste sentido, as oficinas de avaliação participativa, que ocorrem teoricamente a cada 06 meses, e as oficinas de trabalho, cuja frequência é maior, são os espaços onde se propõe a interação entre todos os atores envolvido na construção do ProBUC se dá. Conhecimentos tradicionais e científicos promoveriam, em tese, um diálogo com vistas a uma permanente avaliação do trabalho desenvolvido com base nos resultados alcançados. A capacitação comunitária, uma necessidade verificada no debate teórico deste artigo para que o monitoramento local seja eficaz, estaria, assim, sendo assegurada por meio desta metodologia.

\subsection{Percurso metodológico}

A presente pesquisa, conforme mencionado, procurou avaliar o funcionamento do ProBUC par- 
tindo da opinião e vivência dos comunitários onde está implementado, havendo a complementação da reunião técnica do ano passado. Uma pesquisa qualitativa que procura elencar algumas das principais limitações e potencialidades do programa com o objetivo de apresentar uma sucinta avaliação. Trata-se, ademais, de parte do projeto de dissertação de mestrado intitulado "Limitações e potencialidades do ProBUC para a gestão ambiental do Amazonas" desenvolvida dentro do Programa de Pós-Graduação em Ciências do Ambiente e Sustentabilidade na Amazônia da Universidade Federal do Amazonas.

No período de 10 a 20 de fevereiro de 2014, realizou-se uma viagem às comunidades da RDS do Uatumã com o objetivo de entrevistar moradores de comunidades onde se insere o programa objeto desta pesquisa. Visitaram-se 10 comunidades: Amaro, Bom Jesus, Boto, Cesaréia, Maracarana, Monte das Oliveiras, Nossa Senhora do Livramento, Santa Helena do Abacate, Santa Luzia do Jacarequara e São Francisco das Chagas. No total foram entrevistados 33 moradores, dos quais são 12 monitores. Posteriormente, no período entre 26 de março e 01 de abril de 2014, foram realizadas as entrevistas com comunitários da RDS Uacari, no município de Carauari. As comunidades visitadas foram 12: Bauna, Boa Vista, Bom Jesus, Cachoeira, Mandioca, Monte Carmelo, Morro Alto, Remanso, São José, Toari, Vila Ramalho e Ximboá. Na RDS Uacari localizou-se o monitoramento de jacarés, inexistente na RDS Uatumã. 25 foi o número de entrevistados, sendo 19 monitores.

Em relação à reunião técnica de avaliação do ProBUC, que se deu em outubro de 2013, durante 2 dias, observou-se que sua finalidade consistia em obter dos técnicos presentes sua avaliação sobre o funcionamento do programa, considerando que a maioria presente já trabalhara no mesmo. Recomendações, ao final, foram feitas com o objetivo de aperfeiçoá-lo.

\section{REFERENCIAL TEÓRICO}

\subsection{Do monitoramento convencional ao monitoramento comunitário na perspectiva da gestão de áreas protegidas.}

\section{I.I Evolução do monitoramento comunitário}

No contexto da gestão de áreas protegidas, o monitoramento constitui ferramenta importante, responsável pela identificação e diagnóstico de algo ao longo de um tempo e espaço determinados. Segundo Junior et al (2011, p.11) monitorar é "acompanhar, diagnosticar e identificar tendências de algo ao longo do tempo [...]", permitindo "[...] compreender o estado da biodiversidade, a forma com esta está sendo usada, seus níveis de ameaça e potencialidades [...]"(idem p.11). Em outros termos, consiste na "[....] observação repetida para fins específicos, de um ou mais elementos do ambiente, de acordo com horários pré estabelecidos, no espaço e no tempo, utilizando métodos de coleta de dados comparáveis [...]" (HOCKINGS, 2000, p.3). Desta maneira, o monitoramento da biodiversidade torna-se um aliado da gestão de áreas protegidas, proporcionando informações para o planejamento da gestão e para auxiliar as pesquisas científicas. Esta atividade há aproximadamente quarenta anos vem sendo realizada com mais frequência (GLASSON, THERIVEL e CHADWICK 1994) em decorrência da constatação dos impactos crescentes da ação antrópica sobre os recursos naturais e a biodiversidade. Construiu-se, paulatinamente, o consenso acerca da necessidade de se compreender ao máximo as dinâmicas dos sistemas complexos que organizam os ecossistemas. Por meio do monitoramento, possibilita-se avaliar o estado de um recurso ou sistema, municiando a gestão da área protegida ou especialmente da UC de prováveis eventos (CUNDILL e FABRICIUS, 2009). Em meados dos anos de 1990, a gestão dos projetos ambientais passou a incorporar o monitoramento como essencial (LAMMERTS van BUEREN e BLOM, 1997). Juntamente com o processo de avaliação da gestão das áreas protegidas, as bases conceituais do monitoramento se expandiram expressivamente nos últimos dez anos (CUNDILL e FABRICIUS, 2009).

Como pontuam Garcia e Lescuyer (2008), os sistemas de monitoramento funcionam por meio de aparelhos que incorporam inovações tecnológicas, as quais permitem a observação das mudanças tanto físicas quanto sociais de um ambiente considerado. São usados para armazenar e ordenar informações estratégicas que dizem respeito às consequências negativas e positivas da implementação de projetos. Por esta razão, muito além de se constituírem como fonte de conhecimento da diversidade biológica local para auxiliar a gestão de recursos naturais, os sistemas de monitoramento podem sinalizar para a gestão mudar os objetivos e rumos anteriormente estabelecidos, influindo diretamente na tomada de decisões. No entanto, diversas dificuldades foram detectadas ao se inserir as atividades de 
monitoramento em escalas distintas daquelas para as quais foram inicialmente programadas. Questionamentos sobre a consistência científica foram realizados em relação ao seu uso diário por projetos de gestão de recursos naturais. Percebeu-se, então, que a incorporação das populações locais nos sistemas de monitoramento poderia proporcionar ganhos às suas finalidades por causa do conhecimento local que possuem. Uma importante constatação para esta mudança de posição científica só ocorreu porque

[...] Em alguns casos, as percepções sobre a situação dos recursos naturais são maiores entre usuários do que entre os cientistas. Os produtores percebem com antecedência, pelo contato próximo, os sinais da superexploração, da distribuição restrita e quando o acesso aos recursos é limitado [...] (REBÊLO e PEZZUTI, 2000).

Segundo Sheil e Lawrence (2004), outro fator relevante para a mudança de paradigma da atividade de monitoramento foi a constatação da aceleração da extinção de espécies biológicas pouco investigadas, em diversas partes do mundo, particularmente nas regiões tropicais. Muitos cientistas propuseram, desde então, a incorporação das populações locais ao trabalho de cogestão de áreas protegidas. As populações locais tornaram-se parte do processo de coleta taxonômica, estimativa de espécies biológicas, ampliando a sensibilização social e comunitária para a conservação dos recursos naturais e da biodiversidade. Esta nova metodologia viabilizou políticas locais e nacionais que buscam a redução dos impactos ambientais sobre as áreas protegidas. Neste cenário, o aproveitamento das experiências comunitárias para realização das pesquisas biológicas ocorridas nos países tropicais é uma realidade crescente há pouco tempo. Os resultados destas abordagens são impressionantes. Estas comunidades, inicialmente mais motivadas pela oportunidade de trabalho (o que é escasso), foram fundamentais para o oferecimento de melhores informações sobre a biodiversidade local a um custo menor do que ocorreria numa abordagem científica tradicional. Como exemplos, podem ser citadas as experiências nas florestas de Kinabalu e Sabah, na Malásia. Pesquisadas há mais de um século por botânicos, estas florestas foram incluídas em um projeto que visava aumentar as informações sobre espécies de palmeira entre dez e dezenove anos. Os resultados obtidos foram significativos e mais rápidos por causa desta participação direta dos comunitários locais. O Programa de Inventário Nacional da Biodiversidade da Costa Rica (INBio), por seu turno, introduziu a participação comunitária para a catalogação de aproximadamente três milhões de espécies de insetos, o que foi concluído em treze anos. Na Área de Conservação Guanacaste foram registrados duzentos mil lagartos de três mil espécies em duas décadas com a colaboração indispensável dos comunitários. Esta participação local também foi essencial para a especificação de mil e dez espécies de insetos mastigadores de folhas de cinquenta e nove espécies de plantas lenhosas na Nova Guiné. Tal trabalho consumiu apenas três anos, surpreendentemente.

Danielsen et al (2003), ao abordar criticamente os trabalhos do monitoramento convencional, mencionam duas limitações encontradas: a ausência de objetivos articulados e a negligência sobre a estimação da diversidade biológica. O trabalho de monitoramento, normalmente, exige muitos investimentos. Estes investimentos envolvem a presença de pesquisadores e a utilização de meios eletrônicos muitas vezes caros, os quais devem ser adaptados a cada realidade, comprometendo sua eficácia ao serem empregados em realidades bastante distintas daquelas para as quais foram programados. Neste sentido, Danielsen et al (2007) informam que os países desenvolvidos utilizam largamente o monitoramento realizado por cientistas profissionais para avaliar as mudanças populacionais e orientar a gestão das áreas definidas como de conservação prioritária. Ademais, os autores ressaltam que os resultados do monitoramento devem gerar uma análise sobre os objetivos traçados pelas políticas globais dirigidas para a biodiversidade. Contudo, nos países em desenvolvimento, nos quais se verificam as maiores concentrações de biodiversidade, os custos dos sistemas tradicionais de monitoramento são elevados, inviabilizando seu financiamento pelos orçamentos públicos locais. Nestes países se faz necessário que a gestão das áreas protegidas recorra a abordagens alternativas de monitoramento como o compartilhamento da coleta de dados com as populações locais, majoritariamente rurais, que vivem nestas regiões protegidas. Esta aproximação potencializaria os resultados. Assim, a inovação proveniente do monitoramento comunitário pode representar menor impacto financeiro, além de ampliar o alcance do monitoramento devido ao conhecimento local das espécies possuído pelas populações locais.

\section{I.2 Limites e princípios do monitoramento comunitário}

Presume-se que a inclusão das comunidades locais na gestão das áreas protegidas e atividades de monitoramento é tendência a se propagar, especialmente nos países tropicais ou em desenvolvimento 
(SHEIL e LAWRENCE, 2004). Entretanto, o envolvimento comunitário se relaciona fundamentalmente ao retorno em benefícios sociais e geração de renda (DANIELSEN et al, 2007), pois não se pode ignorar que a qualidade de vida é uma das condições importantes para o empenho pela conservação ambiental. Há autores que veem nesta modalidade de monitoramento a sua importância no fato de que ela pode ser alternativa aos programas governamentais tradicionais dos países em desenvolvimento que apresentam elevada corrupção. Neste contexto, a participação comunitária reduziria incidência de malversação de recursos, apesar da lacuna de estudos comprobatórios a respeito (AFRIDI, 2008). Observam, no entanto, Jacoby et al (1997) que o monitoramento comunitário também possui limitações. Um elemento estaria associado à própria qualidade dos dados coletados, sobretudo nos momentos de registro e tratamento dos dados coletados no sistema formal de monitoramento. Esta dificuldade ressaltada exige permanente capacitação dos comunitários.

A maioria dos sistemas comunitários de monitoramento é financiada por agências internacionais, especialmente organizações não governamentais. Este processo é estimulado pela descentralização da gestão dos recursos naturais a fim de incluir, sobretudo, as populações locais. Esta proposta pretende ser hegemônica, pois vislumbra maior abrangência e profundidade para o desenvolvimento do monitoramento (GARCIA e LESCUYER, 2008). Entretanto, conforme Whitelaw et al (2003), muitas vezes a inconsistência de recursos financeiros dos doadores e o descumprimento de prazos da parte dos gestores promove desinteresse entre comunitários.

Quatro razões são apresentadas para o rápido crescimento do monitoramento comunitário em termos internacionais: a) reduzida capacidade dos governos para monitorar os ecossistemas, em função dos cortes orçamentários expressivos para programas e atividades ambientais; $b$ ) atividades de monitoramento realizadas pelos governos findam inadequadas para abordar questões ambientais complexas e emergentes; c) reconhecida necessidade de envolver as partes interessadas e os cidadãos em processo de planejamento e gestão; d) desejo dos comunitários em adentrar neste processo. A parte educacional desta inovação está justamente nesta responsabilidade que os comunitários assumem para a gestão da conservação dos recursos naturais (WHITELAW et al, 2003, p. 412). Esta inovação metodológica também é fruto do aprofundamento do debate global acerca da necessidade de sistemas participativos e de avaliação de iniciativas voltadas para o desenvolvimento. As experiências de monitoramento comunitário se disseminaram nas últimas duas décadas. Estão em processo de construção, requerendo o envolvimento de todos os comunitários para que realmente dêem resultados. Para Gaventa, Creed e Morrissey (1998) aspectos como negociação, resolução de conflitos e empoderamento comunitário são vitais para o desempenho do monitoramento comunitário. A operacionalização dos sistemas de monitoramento comunitário possui 5 (cinco) princípios:

Tratam os bens e serviços que a comunidade origina do ecossistema que está sendo monitorado; Os benefícios para as populações locais excedem os custos;

Conflitos políticos entre gestores governamentais e as comunidades não limitam o envolvimento dos atores locais;

Os dados são arquivados, analisados e acessíveis localmente;

$\mathrm{O}$ monitoramento baseia-se em instituições tradicionais existentes e outras estruturas de gestão, tanto quanto possível. No entanto, o sistema pode não funcionar corretamente quando a necessidade de melhor governança em contraposição a práticas tradicionais e antidemocráticas. (DANIELSEN et al, 2005 apud GARCIA e LESCUYER, 2008).

Pelos princípios expostos, pode-se certificar que a participação dos comunitários não deve ser apenas na execução do trabalho de pesquisa, mas na capacidade de retornos que poderão ser convertidos em benefícios para os próprios comunitários. Problemas políticos, em certas ocasiões, são verdadeiros óbices ao funcionamento adequado de programas inspirados nesta metodologia. Em contextos onde mudanças são difíceis de acontecer, marcadas por péssimas relações entre comunitários e autoridades públicas e, sobretudo, onde as necessidades coletivas em termos de saúde, educação, geração de renda, entre outras, são significativas, a propensão de programas e sistemas participativos para a gestão de áreas protegidas fracassarem é maior.

O monitoramento comunitário parece condizente com a realidade amazônica. De fato, os baixos investimentos dos governos locais em ciência e tecnologia dificultam o monitoramento convencional, favorecendo, por outro lado, o surgimento de oportunidades para o monitoramento comunitário. No 
entanto, cabe questionar até que ponto os interesses dos próprios comunitários estão sendo considerados neste processo. Esta proposta poderia também estar facilitando que os governos se desobriguem de se responsabilizar diretamente através de seus orçamentos do investimento em monitoramento.

\section{RESULTADOS E DISCUSSÕES}

Dentre as limitações apontadas pelos teóricos do monitoramento comunitário e confirmadas pela realidade apresentada durante as atividades de campo da presente pesquisa, destaca-se que os benefícios sociais e econômicos gerados com o auxílio do monitoramento comunitário estão muito aquém de seus potenciais. Na verdade, não se constatou uma relação de complementaridade entre os dados coletados pelos monitores comunitários do ProBUC e retornos por meio de políticas públicas e atividades de manejo para as comunidades envolvidas.

A dependência de financiamento externo, mesmo sendo uma tendência mundial como afirmado anteriormente, não deixa de gerar dúvidas sobre as intenções subjacentes ao programa de monitoramento. Esta condição influencia as prioridades do programa em análise, pois quem financia geralmente impõe suas prioridades para o desenvolvimento da atividade, trazendo implicações éticas e políticas fundamentais, como salientam alguns autores (DANIELSEN et al, 2005; GHATE e NAGENDRA 2005; GARCIA e LESCUYER, 2008). Não se sabe até quando a Fundação norte-americana Moore continuará financiando o ProBUC. A maioria dos comunitários ignora esta condição. Identificam o ProBUC tão somente como um programa governamental que deve beneficia-los dentro do contexto da unidade de conservação em que vivem. Alguns deles souberam durante os períodos em que o programa ficou paralisado que a razão teria sido a falta de recursos. Porém, não lhes foi explicado que tal ocorrência derivava de momentos em que os convênios firmados entre esta instituição patrocinadora e o governo estadual estavam suspensos. Nem mesmo sua concordância a respeito foi considerada na ocasião em que o governo mencionado optou pelo aporte de financiamento externo para desenvolver esta modalidade de monitoramento.

A falta de comunicação entre o programa e os comunitários, bem como a dificuldade destes próprios moradores locais em definir os objetivos do ProBUC, sugerem que a valorização do conhecimento empírico e o suposto empoderamento não estão satisfatórios, o que, em última análise, compromete a propalada participação comunitária. Afinal, os atores locais precisam ser protagonistas nas principais decisões para que possam efetivamente incorporar com maior clareza a responsabilidade pela conservação da biodiversidade, como um dos princípios elencados no debate teórico deste artigo. Os boletins anuais distribuídos pelos técnicos do programa, mostrando os resultados do trabalho de monitoramento, revelam-se insuficientes para o conhecimento dos comunitários. Ou seja, os comunitários protagonizam o monitoramento, mas não conhecem seus resultados. Não conhecem a conclusão de seu próprio trabalho. $\mathrm{E}$, por conta disto, vivem numa permanente expectativa a respeito do que possa retornar em benefícios para sua vida a partir do trabalho de monitoramento realizado.

Considerando o princípio citado sobre arquivo, análise e acesso local de dados, constata-se o quanto o ProBUC fica a desejar. Trata-se de um de seus principais obstáculos. A falta de conhecimento de sua abrangência e do domínio das informações geradas pela coleta de dados até o momento faz com que não se identifique com precisão as áreas de potencialidades para atividades econômicas e áreas de escassez que poderiam ser preservadas. Esta ausência de conhecimento se refere tanto aos técnicos do programa quanto aos próprios comunitários. Seria essencial para atualizar os planos de manejo, no caso das unidades de uso sustentável. A ausência de recursos tecnológicos, por sua vez, dificulta a obtenção de dados mais precisos sobre o que é monitorado, resultando em baixa qualidade de dados. É inconcebível que o fato de um programa de monitoramento ser realizado por comunitários deva prescindir dos meios mais atualizados e disponíveis para subsidiar este trabalho, como se este direito fosse exclusivo do monitoramento convencional. Esta limitação articula-se à necessidade de um processo de alfabetização que permita aos monitores a capacidade de registrarem com segurança os dados obtidos por seu trabalho.

A inexistência de corpo técnico adequado e permanente na gerência de funcionamento do programa é outra limitação que precisa ser superada por meio de concurso público que crie uma carreira profissional que possa oferecer dedicação ao funcionamento deste programa de monitoramento com menos interferências políticas dos gestores momentâneos. Observou-se no debate teórico sobre 
monitoramento comunitário que o descumprimento de determinadas obrigações pelos gestores invariavelmente conduz ao desânimo dos comunitários. Esta realidade pode e deve ser evitada à medida que se garantir maior independência funcional dos técnicos do programa. Desta maneira, estariam menos imunes ao humor do gestor que não cumprisse as metas e o planejamento que venham a ser definidos para o programa.

O resgate da participação do ProBUC é fundamental para sua revitalização. Trata-se de um potencial a ser devidamente valorizado. De acordo com os princípios mencionados, os conflitos políticos não podem impedir a participação comunitária, assim como a existência de práticas antidemocráticas podem comprometer a legitimidade das decisões tomadas. Por esta razão, a participação deve acontecer com maior frequência e não se limitar aos técnicos e monitores do programa. Muitos comunitários reclamaram que técnicos do programa apenas dialogam com os monitores e não mais com a comunidade de um modo geral, quando realizam a manutenção do programa, a cada 2 meses, em média. Nestas ocasiões, os técnicos do ProBUC ou o gestor da UC recolhe os formulários preenchidos pelos monitores e entrega os novos. Muitos comunitários nem tomam conhecimento da ocasião em que o técnico comparece. Neste sentido, há de se proporcionar espaços permanentes de participação ampla de comunitários e pesquisadores com o fito de identificar as limitações e propor mudanças que visem o aperfeiçoamento do ProBUC.

A adaptação é uma potencialidade própria dos programas de monitoramento comunitário. Há de se avaliar sempre se os componentes de monitoramento de cada UC estão de fato correspondendo às necessidades e interesses locais. Aparentemente, pelo depoimento dos comunitários entrevistados e pelas observações dos técnicos na reunião citada, os componentes estão em conformidade com as prioridades locais. Porém, a repetição dos componentes nas UCs pesquisadas pode ser também um indício de que espécies e recursos importantes que poderiam mobilizar mais comunitários em torno do ProBUC podem ter sido descartados. Esta questão exige uma investigação mais detalhada.

Sem desmerecer o diagnóstico supracitado, entende-se que a dificuldade maior de implementação do programa é de ordem estrutural: sua desarticulação com os demais programas e políticas desenvolvidos no interior das UCs em que se encontra. É necessária uma reinserção deste programa amazonense de monitoramento a partir de uma estratégia de desenvolvimento local, na qual ele contribua com o fornecimento de informações aptas para atividades de manejo previstas em cada UC de usos sustentável e de outras formas possíveis de inserção comunitária na unidade de proteção integral. Não há, portanto, como exigir empenho dos comunitários se não houver iniciativas que melhorem sua qualidade de vida em paralelo ao trabalho de conservação ambiental. Destaque-se também que os comunitários residentes das UCs onde está implantado precisam ser considerados em permanentes processos avaliativos do ProBUC.

\section{CONCLUSÃO}

A incorporação do monitoramento ao processo de avaliação de gestão de áreas protegidas em nível mundial reconheceu paulatinamente que as comunidades locais poderiam contribuir significativamente com esta função graças ao seu conhecimento empírico sobre o uso dos recursos naturais e a preservação da biodiversidade. Assim, o monitoramento comunitário ganhou relevância nos países tropicais, onde os investimentos no monitoramento tradicional tornaram-se raros por conta de seu elevado custo. $\mathrm{O}$ monitoramento comunitário, mais democrático que o antecessor, por outro lado, apresenta algumas limitações, como a qualidade de dados e a necessidade de retornos sociais e econômicos aos comunitários para que haja uma efetiva participação local. Em contextos de elevada pobreza, falta de serviços públicos adequados e baixa participação política, esta modalidade de monitoramento de viés participativo apresenta entraves para se consolidar. Ademais, o fato de os recursos que o financiam serem, de um modo geral, advindos de organismos internacionais faz com que questionamentos que estejam por trás de sua implantação sejam suscitados. No Amazonas, a vigência do programa de monitoramento comunitário ProBUC não foge à regra. Implantado no período de 2005 a 2010 em três UCs estaduais, ele se destina a auxiliar a gestão das mesmas com o fito de proteger a biodiversidade e utilizar os recursos naturais sustentavelmente. A participação comunitária, principal potencial deste programa amazonense, pelos resultados obtidos, não está à altura da profundidade necessária que identifique os moradores locais com a inserção do programa. Muitas fragilidades foram 
evidenciadas pelos comunitários entrevistados e no evento avaliativo com técnicos que já trabalharam no programa, como dependência financeira de uma fundação norte-americana, ausência de um corpo técnico permanente e suficiente, desorganização de dados, entre outras limitações. As recomendações traçadas também são limitadas como propostas para resolver os gargalos. Outras avaliações precisam acontecer com frequência, incluindo com os comunitários envolvidos para se corrigirem as falhas e se superarem as lacunas. Faz-se mister que o governo estadual seja capaz de promover uma articulação política que insira o ProBUC como prioridade na agenda de prioridades no âmbito da gestão das UCs estaduais. Ou esta tarefa será assumida ou dificilmente o ProBUC se consolidará como alternativa de monitoramento comunitário amazônico, capaz de se irradiar às demais UCs que compõem o sistema estadual de unidades de conservação e na Amazônia de um modo geral. Fundamental se faz que uma boa gestão do ProBUC é condição para que auxilie a gestão das UCs estaduais em que se insere.

\section{REFERÊNCIAS}

ABBOT, JoanneGUIJT, Irene. Changing Views on Change: Participatory Approaches to Monitoring the Environment. In: International Institute for Environment And Development, Londres, UK, 1998. Disponível; em: <http://books.google.com.br/books?hl=pt-BR\&lr=\&id=ZeL0o4AF8aAC\&oi=fnd\&pg=PA4\&ots=XT85gftYu\&sig=1jecuGKrhhHiyO8Dts3RvJGQtkI\#v=onepage\&q\&f=false>. Acesso em 03. Jan. 2014.

AFRIDI, Farzana. Can community monitoring improve the accountability of public officials? In: Economic e political weekly. Vol. 43. N. 42, 2008. Disponível em: <http://www.jstor.org/stable/i40010942> Acesso em 11. Nov. 2013.

BATISTA, Gelson da Silva et al. O uso do recurso pesqueiro na reserve de desenvolvimento sustentável de Uacari, Amazonas, Brasil, subsídios para elaboração do plano de gestão. In: CALANDINO, D.et al. (orgs.). Áreas protegidas da Amazônia. Brasília, DF. Ministério do Meio Ambiente, 2008.

BORGES, Sergio Henrique et al .Construindo a gestão do parque estadual Rio Negro setor norte, Amazonas, Brasil.In: In: CALANDINO, D. et al. (orgs.). Áreas protegidas da Amazônia. Brasília, DF. Ministério do Meio Ambiente, 2008.

CUNDILL, George; FABRICIUS, Christo. Monitoring in adaptive co-management: towards a learning based approach. In: Journal of environmental management, n. 90, issue 11, 2009. Disponível em: < http://www. sciencedirect.com/science/article/pii/S0301479709001510> Acesso em 27 set. 2013.

DANIELSEN, F. et al. Biodiversity monitoring in developing countries: what are we trying to achieve? Oryx, 2003 - Cambridge Univ Press.

et al; Monitoring matters: Examining the potential of locally-based approaches. In: Biodiversity and conservation v. 14 Issue 11, pp 2507-2542, Springer, 2005. Disponível em: < http://link.springer. com/article/10.1007/s10531-005-8375-0\#page-1> Acesso em 14 jan. 2014

et al. Increasing conservation management action by involving local people in natural resource monitoring. In: AMBIO: journal of the human environment, v. 36, n. 7, 2007.

DAVIES, Rick. An evolutionary approach to facilitating organisational learning: an experiment by the Christian Commission for Development in Bangladesh. In: Impact Assessment and Project Appraisal, vol. 16, n. 3 , 1998, Beech Tree Publishing, I 0 Watford Close, Guildford, Surrey GUI 2EP, UK. Disponível em: <http:// www.tandfonline.com/doi/pdf/10.1080/14615517.1998.10590213> Acesso em 13 jan. 2014.

EVANS, Kristen; GUARIGUATA, Manuel R. Participatory Monitoring in tropical forest management: a review of tools, concepts and lessons learned. Center for International Forestry Research, 2008, Bogor, Indonésia. Disponível em: < http://www.cifor.org/publications/pdf_files/Books/BGuariguata0801.pdf> Acesso em 14 jan. 2014 
GARCIA, Claude A.; LESCUYER, Guillaume. Monitoring, indicators and community based forest management in the tropics: pretexts or red herrings? In: Biodiversity and Conservation, 2008 - Springer. Disponível em: <http://link.springer.com/article/10.1007/s10531-008-9347-y> Acesso em 13 jun. 2013.

GAVENTA, John; CREED, Victoria; MORRISSEY, Janice. Scaling up: participatory monitoring and evaluation of a Federal Empowerment Program. In: New Directions for Evaluation, n. 80, Winter, 1998. Disponível em: <http://onlinelibrary.wiley.com/doi/10.1002/ev.1119/abstract> Acesso em 12 set. 2013.

GHATE, Rucha; NAGENDRA, Harini. Role of monitoring in institutional performance: Forest management in Maharashtra, India. In: Conservation and society, v. 3, issue 2 pp. 509-532, 2005. Disponível em: < http: / conservationandsociety.org/article. asp?issn $=09724923$; year $=2005$; volume $=3$; issue $=2$; spage $=509$; epage $=532$; aulast $=$ Ghate $>$ Acesso em 14 jan. 2014

GLASSON, Jhon; THERIVEL, Riki; CHADWICK, Andrew. Introduction to environmental impact assesment. In: Introduction to environmental impact assessment. The natural and built environment series, fourth edition. UCL Press, Londres, 1994. Disponível em: <http://books.google.com.br/books?hl=ptBR\&1$\mathrm{r}=\& \mathrm{id}=$ NefZAAAAQBAJ\&oi=fnd $\& \mathrm{pg}=\mathrm{PP} 1 \& \mathrm{dq}=.+$ Introduction+to+environmental+impact + assesmen $\mathrm{t} \&$ ots $=\mathrm{dnHMQq8F} \_$Y\&sig $=5$ uioBsaEifMJQli6VehxHXo2jF8\#v=onepage\&q=.\%20Introduction $\% 20 \mathrm{to} \% 20$ environmental\%20impact\%20assesment\&f=false> Acesso em 10 jan. 2014.

HOCKINGS, Marc. Evaluating effectiveness - A framework for assessing the management of protected areas. In: Oxford journals, v.53, n.9, 2000. Disponível em:< http://bioscience.oxfordjournals.org/content/53/9/823.short> Acesso em 27 dez. 2013.

JUNIOR, Sinomar F. Fonseca et al. Programa de monitoramento da biodiversidade e do uso de recursos naturais- ProBUC: a experiência das unidades de conservação estaduais do Amazonas. Manaus, Centro Estadual de Unidades de Conservação, 2011.

LAMMERTS van BUEREN EM, BLOM EM. Hierarchical framework for the formulation of sustainable forest managemente standards. In: Tropenbos Foundation, 1997. Disponível em: < http://www.piec.org/ pathfinder/Pathfinder_portal/Instruments_Engl/A3Hierarchical_framework/print/CI_framework_PF.pdf>. Acesso em 23 nov. 2013.

REBELO, George; PEZZUTI, Juarez. Percepções sobre o consumo de quelônios na Amazônia: sustentabilidade e alternativas ao manejo atual. Ambient. soc., Campinas, n.67, jun. 2000.Disponívelem <http://www. scielo.br/scielo.php?script=sci_arttext\&pid=S1414753X2000000100005\&lng $=$ pt\&nrm=iso $>$. Acesso em 25 set. 2013.

SHEIL, Douglas; LAWRENCE, Anna. Tropical biologists, local people and conservation: new opportunities for collaboration. In: Trends IN Ecology and Evolution, vol. 19, n. 12, 2004. Disponível: < http://www.sciencedirect.com/science/article/pii/S0169534704002915> Acesso em 27 set. 2013.

STUART-HILL, Greg et al. The event book system: community-based monitoring in Namibia. In: Biodiversity and Conservation, v. 14, pp. 2611-, 2631, Springer, 2005. Disponível em: < http://link.springer.com/article/10.1007/s10531-005-8391-0\#page-1> Acesso em 14 jan. 2014.

WHITELAW, Graham, et al Establishing the Canadian community monitoring network. In: Environmental monitoring and assessment, 88, 2003. Disponível em: <http://link.springer.com/article/10.1023/A:1025545813057\#page-1>Acesso em 30 nov..2013. 